\title{
Study on the Key Technology of Personalized Recommendation of Case-based Reasoning
}

\author{
Fuliang $\mathrm{Li}^{1}$, Jieli $\mathrm{Sun}^{2}$ and Xia Zhangi ${ }^{3}$ \\ ( 1,3 Hebei University of Economics and Business Economics and Management \\ College , Hebei Province Shijiazhuang City 0500912 Hebei University of \\ Economics and Business Information and Technology College Hebei Province \\ Shijiazhuang City 050061) \\ lifuliang@heuet.edu.cn
}

\begin{abstract}
The key technology of personalized recommendation based on CBR involves the representation and the organization of case, construction and maintenance of multiple cases library, judging of the similarity of case and methods of retrieval, and the combination of personalized recommendation technology. The four interrelated aspects are the important links to design the personalized recommendation system. This paper studies the key technology of the personalized recommendation system based on CBR.
\end{abstract}

Keywords: personalized recommendation; CBR (case-based reasoning); combination recommendation technology

\section{Introduction}

The study on personalized recommendation system of Case-based reasoning involves four interrelated aspects: the representation and the organization of the personalized recommendation on case-based reasoning, construction and maintenance of multiple cases library of personalized recommendation, judging of the similarity of personalized recommendation case and methods of retrieval, and the combination of personalized recommendation of Case-based reasoning.

Personalized case representation and case organization are the foundation of the building and maintenance of personalized recommendation of the casebase, and the preconditions for case-based reasoning. The building and maintaining of multiple cases library ensures the success of the application of personalized recommendation system; determination of case similarity and search is for the personalized recommendation library, and they are guarantees for the success of personalized recommendation of the Case-based reasoning. These four parts coordinate with each other, jointly complete calculation output of the results of the personalized recommendation.

The traditional recommendation technology based on collaborative filtering technology produces results on the user's dominant score, which is not only inconvenient to use, but unable to produce an effective solution to such problems as the sparse data problem in Collaborative Filtering Recommendations and cold start.

According to the author's research, most personalized recommendations, on the needs and characteristics of the user, are the calculations on the result of personalized recommendation with the unified personalized recommendation algorithm provided by personalized recommendation system. Without the choices on the corresponding personalized recommendation of the system parameters for personalized customer demand, it can not provide different personalized recommendation strategy for different users. 
Case-based reasoning Personalized recommendation system did not involve too much about the automatic access to the case, generation technologies and the casebase building. There is not enough study about how to get the data of the user about his personalized information on the case, how to take effective measures to optimize case show and how to do case organization and index in different applications when Case-based reasoning methods be used in personalized recommendation system.

\section{Theory Related to Case-based Reasoning Personalized Recommendation Systems}

The main points about Case-based reasoning Personalized Recommendation system have been divided into three parts: first, how to add Case-based reasoning approach to Personalized Recommendation System Design and Implementation; Second, how to apply presentation of the Personalized recommendation and case design organizations to personalized recommendation system; the third, how to achieve the determining of the Similarity case index of personal recommendation case with case-based reasoning methods, and how to design Case-based reasoning personalized recommendation algorithm and apply it to the process of Personalized recommendation system.

\subsection{Main Content of Case-based Reasoning}

Case-based reasoning is a method of reasoning to solve the current similar problems by analogy and associations with previous experience and methods. With the deepening of the case-based reasoning method, many application fields have been involved in the study of case-based reasoning. In solving problems, there are times when it is difficult to abstract rules to present it, then solution can be provided for the current based on the previous experience, therefore, Case-based reasoning and case-based reasoning methods of research have been carried out continuously and applications have become increasingly extended. At present, case-based reasoning research mainly focuses on the following aspects.

Study on the process of case-based reasoning and case-based reasoning system design. The following studies can be included in designing problems of Case-based reasoning system: the process of case-based reasoning model, the presentation and the organization approach of case, case retrieval technology, library construction and maintenance of case technology, how to extract automatically from the structure of non-text case the property features of the case, technical amendments of the case and the access method to the amendments rules, acquisitions of distributed case, as well as management and maintenance of distributed database.

Research on application of case-based reasoning system in the relevant fields, Overcoming the difficulties in acquisition of knowledge on the traditional rule-based reasoning systems and the vulnerability of reasoning, Case-based reasoning approach has been widely used in a variety of applications, such as Case-based reasoning information retrieval, personalized recommendation, machine learning, knowledge discovery, data mining and decision support systems

\subsection{The Model of Main Case-based Reasoning Process}

On the analysis and summary of related research literature on CBR, several models of CBR reasoning process have been put forward, and the Comparatively influential are mainly the following four models: CBR process model put forward by Hunt, the CBR process model proposed by Leake, the CBR cycle R4 reasoning process model proposed by Aamodt and Plaza, and R5 reasoning model raised by Gavin Finnie and others. R4 the reasoning process model of CBR and R5 reasoning model of CBR are mainly discussed here. 


\subsection{Commonly Used Personalized Recommendation Technology}

At present, the personal technology commonly used in the recommended system is the followings: collaborative filtering based recommendation, content-based recommendation, knowledge- based recommendation, Demographic-based recommendation and Utility-based recommendation

Collaborative Filtering: Collaborative Filtering is to recommend resources based on the similarity of Users obtained by evaluation collection of the users on projects recommended.

Content-based Recommendation: Content-based recommendation is to learn the user's interest by the characteristics of objects evaluated, to recommend resources on the degree of their match between the user' $\mathrm{s}$ date and the forecasting project and by the comparison of user profile and resources recommended project.

Knowledge-based Recommendation: Knowledge-based Recommendation has the knowledge about specific commodity meeting the specific needs of the user and thus derives the correlation between commodities recommended and user' $\mathrm{s}$ demand. Knowledge-based recommendation makes recommendation for user's need derived from the storage of knowledge in recommendation system.

Demographic-based recommendation: Demographic-based recommendation makes recommendation on the user statistics. It classifies the users by their personalization features and then recommend under the category of users.

Utility-based recommendation Utility-based recommendation makes calculations on effectiveness of the project when used, the core is how to create utility function for every user and consider non-product attributes such as the reliability of provider and availability of products.

Collaborative filtering has a wide range of applications as a typical personalized recommendation technology, and its advantage is to find new interested information for the user, its main problems lie in sparse, cold start, a special user and scalability. The advantages of content-based recommendation are its simplicity and effectiveness, but it is unable to distinguish between quality and style of resources only finds the resources similar to the known interest of the user. The collaborative filtering recommends resources by the user's similarity, comparing the user's profile instead of profile of resources and user.

The emphasis and difficulty in Knowledge-based recommendation is to acquire knowledge. Due to the small requirements to users, Knowledge-based recommendation is applicable when user browses temporarily and randomly. Demographic-based recommendation seems similar to Collaborative filtering, but the actual use of the data is completely different. The advantage of the Demographic-based recommendation is that it does not require historical date evaluation of the user, while content-based recommendation and collaborative recommendation technology require historical data. Utility-based recommendation is mostly applied in personalized recommendation on ecommerce. The advantages are that non-product factor can be considered in some utility functions, such as the vendor's reliability, product availability.

\section{The Key Technologies on Personalized Recommendation of System in Case-based}

The study on personalized recommendation system of Case-based reasoning involves four interrelated aspects: the representation and the organization of the personalized recommendation on case-based reasoning, construction and maintenance of multiple cases 
library of personalized recommendation, judging of the similarity of personalized recommendation case and methods of retrieval, and the combination of personalized recommendation of Case-based reasoning

Personalized case representation and case organization are the foundation of the building and maintenance of Personalized recommendation of the casebase, and the preconditions for case-based reasoning. The building and maintaining of multiple cases library ensures the success of the application of Personalized recommendation system; determination of case similarity and search is for the personalized recommendation library, and they are guarantees for the success of personalized recommendation of the Case-based reasoning. These four parts coordinate with each other, jointly complete calculation output of the results of the personalized recommendation.

\subsection{The Representation and the Organization of Case}

To find an appropriate representation of the three parts is the first problem to solve for $\mathrm{CBR}$, that is, to determine the appropriate characteristics of the case to represent $\mathrm{t}$. The following questions are often involved in case representation: how to define the relevant application of cases; how to create descriptions and use of case; what kinds of data to store in system cases; how to choose the right data to describe the structure of specific problems.

For different CBR systems, different Case characteristic attributes and representations are required. In the recent study, a lot of CBR system uses complex case representations to describe the conceptualized case. That case is closely related to specific applications and therefore case representation of personalized recommendation in case-based reasoning system needs to analyze and make use of the representation of personalized recommendation system. collaborative filtering on the user's score, GroupLens, is represented by the user-based - the project evaluation matrix, Content-based personalized recommendation system Personal Web Watcher is demonstrated by Key words weighted vector space model, combination-based personalized recommendation system $\mathrm{Fab}$ is shown by vector space model; the vector space model is currently the most widely-used method to show the user model. The case organization is an important part in the design of the CBR system, so the quality of its strategy will greatly influence the efficiency of CBR system.

\subsection{Construction and Maintenance of Casebase}

All cases composed of a collection of the Casebase. As an essential part in CBR, and the most important part of knowledge base in the case-based reasoning Casebase involves historical experience in application field; the form of organization of casebase and case representation are closely associated. In 2003, Finnie and others put forward a process of reasoning the R5 model of CBR, and the building of casebase was absorbed into the main reasoning process of case-based reasoning.

For the complex application systems, the casebase may be very large, but the speed of retrieval cases can be increased by the establishment of a multi-level index for casebase. The establishment of casebase is the process of constant improvement and updating and achieving its maintenance by continuous learning. The main purpose is to restrict the size of the casebase but maintain its performance. The main task of casebase maintenance is to keep the representative of case in base, extend the coverage of the case in the casebase and to reduce the redundancy. In 2003, when NI Zhi-wei and others were given improved strategies to maintain the program to delete, from another point of view, they proposed maintenance strategy based on case increasing to ensure the performance of the system will not be affected. 


\subsection{Similarity Determining and Search of Case}

Case similarity is level of degree of property value of the issue and property value in the past cases, including numerical value and character data. The commonly used calculation model in calculating the case similarity are the following three:

The Nearest Neighbor Method: The nearest neighbor method believes attribute Set between two cases is the same, and so does the weight. So when used, the case representation and the give of case attributes and weight is comparatively simple.

TC Similarity Law: TC similarity law applicable is suitable to the field where the property can be expressed in binary.

\section{Improving of TC Similarity Law}

\subsection{Personalized Combined Technique Recommended}

Case-based reasoning approach has provided a good basis for personalized recommendation system, but the results of personalized recommendation still need the help of the relevant theory on personalized recommendation. To ensure a relatively precise recommendation in real-time conditions, the researchers put forward a wide range of recommendation technology and algorithms, but with deepening research and analysis on the related personalized recommendation technology and algorithm. Because of its own limitations, there are always some shortcomings and deficiencies. For all kinds of problems on recommendation technology, Hybrid Recommendation can be applied in practical application, to make up shortcomings of all kinds recommendation technologies. Hybrid Recommendation is an important principle, avoiding or making up for the weaknesses of recommendation technology after the combination

At present, the combination of content-base recommendation and Collaborative filtering is the mostly studied and applied Hybrid Recommendation. The combination of the two recommended techniques in Content-based and collaborative filtering can overcome some of their shortcomings. To overcome sparse nature in collaborative filtering, the content of resources having browsed can be used to expect the user's evaluation on other resources, which will increase the density of the resource assessment, and based on these recommendation, collaborative filtering recommendation can be made so as to enhance the function of collaborative filtering.

No matter how to combine the content-based recommendation and collaborative filtering recommendation, there always exists the problem of initialization, because they require the historical evaluation of the users. But with the introduction of case-based reasoning technology issues as initialization can be effectively resolved.

\section{Conclusion}

Case-based reasoning personalized recommendation system is the bridge between the information users and information services systems for a smooth transfer of demand and services, is platform where information service system provides information exchange and data processing. The quality of personalized recommendation system design determines the timely and accurate transmission of information between the user and information services, determines the level of information services and the interests of both sides are also directly related. This article mainly discusses related theory on personalized recommendation systems of the case-based reasoning. Based on the analysis of the relevant theories, technologies and applications, it summed up the main direction of research on personalized recommendation systems of the case-based reasoning and put forward the key technologies in personalized recommendation of case-based reasoning system. 


\section{Acknowledgments}

This work is supported by the research and development of science and technology plans project sustentation fund of Hebei Province (No. 14457419D)

\section{References}

[1] J. Schafer, J. Konstan, and J. Riedi, "Recommender systems in e-commerce", EC'99:Proceedings of the 1st ACM conference on Electronic commerce., (1999), pp. 158-166.

[2] J. B. Schafer, J. A. Konstan, and J. Riedl, "E-commerce recommandation application", Data Mining and Knowledge Discovery, vol. 5, (2001), pp. 115-153.

[3] T. Zhou, LL. Jiang and R. Q. Su, "Effect of initial configuration on network-based recommendation [J]", EurophysLett, vol. 81, (2008), pp. 58004.

[4] Z K. Zhang and P. Wu, "Enhancing personalized recommendation on weighted social tagging networks [J]”, Physics Procedia, vol. 3, (2010), pp. 1877-1885.

[5] R. J. Mooney and R. Loriene, "Content-based book recomending using learning for text categorization", In Proceedings of 5th ACM Conference on Digital Libraries, (2000), pp. 195-204.

[6] D. W. McDonald, "Ubiquitous Recommendation Systems", IEEE Computer Magazine, vol. 10, (2003), pp. 111-112.

[7] Z K. Zhang, T. Zhou and Y C. Zhang, "Personalized Recommendation via Intergrated Diffussion on User-Item-Tag Tripartite Graphs \{J\}”, Physica A, vol. 389, (2010), pp. 179-186.

[8] Z. Huang, H. Chen, and D. Zeng, "Applying associative retrieval techniques to alleviate the sparsity problem in collaborative filtering”, ACM Trans.on Information Systems, vol. 1,(2004), pp. 116-142.

[9] L. Zhao, N. J. Hu, and S. Z. Zhang, "Algorithm design for personalization recommendation systems", Journal of Computer Research and Development, vol. 8, (2002), pp. 986-991.

\section{Author}

Fuliang Li, male, associate professor, he is mainly engaged in data mining and Internet Application Research. 